\section{Picturing pathology}

\author{
By Tracey Baas, Senior Editor
}

A Stanford University team has developed a computational pathology system called C-Path that provides more accurate prognostic scores of breast cancer tissue than classical pathology. ${ }^{1}$ The next steps are modifying the method to handle whole-tissue slide samples and seeing how it performs in a prospective, multicenter trial.

Traditionally, pathologists visually characterize the type and aggressiveness of breast cancer using only three cellular features-the percentage of tumor made up of tubular structures, the diversity of nuclei in cells and the frequency of cell division-and assign prognostic scores based on a scale first proposed in the 1920s. Identifying tissue types as skin, duct or organ lining (the epithelia) or connective tissue (the stroma) is an important part of the grading system.

A Stanford group led by Daphne Koller, professor of computer science, hypothesized that computer-aided analysis of digital images of tumor samples might identify new, clinically relevant cellular features and more accurately predict patient outcomes.

The first step was getting C-Path to differentiate stromal regions from epithelial regions of breast cancer samples. To do so, the group used digital images from breast cancer tissue microarrays obtained from patients with a known prognosis.

The team used image processing techniques to define a range of features that characterize the epithelial regions and stromal regions. The group also included many features that describe the more global, spatial structure of the tumor. Altogether, the researchers created a 6,642-feature set, the majority of which had not been previously used in analysis of pathology samples.

Next, the researchers used the known patient prognoses and the 6,642-feature set to construct C-Path such that it learned a scoring system predictive of patient outcome. As proof of concept, the group used C-Path to analyze digital images from a second cohort, obtained from a different hospital and a different patient population. The scores were significantly associated with overall survival $(p=0.001)$.

$C$-Path evaluated data from breast cancer tissue microarrays containing sample cores that were $0.6 \mathrm{~mm}$ in diameter. Pathologists graded the same tissue microarray images as C-Path and found no significant association with survival $(p=0.4)$. Pathologists typically use a microscope to study larger tissue samples fixed on a glass slide.

Further computational analysis showed only 11 of the 6,642 features -8 epithelial and 3 stromal-significantly contributed to the model's scoring system. In addition, the stromal features were a stronger predictor of patient outcomes than the epithelial features.

"A particularly interesting aspect of their study was that they found stromal features to be more predictive than epithelial features," said Gerardo Fernandez, medical director for digital pathology at Roche's Ventana Medical Systems Inc. unit. "Pathologists have been focusing on the same three microscopic epithelial features for almost a hundred years. Koller's study suggests that some of the stromal features might be equally informative."

Data were published in Science Translational Medicine. The work is currently unpatented and is available for licensing from Stanford University. C-Path was built to work on Definiens AG's Developer XD image analysis platform.

\section{Sample size}

Despite C-Path's improved prognostic ability versus the naked eye, lead author Andrew Beck does not think pathologists are headed for extinction.

"I see the field of pathology thriving as more and more biological and computational tools are developed to extract larger and larger amounts of data from tissue samples," said Beck, who now is an assistant professor of pathology at Harvard Medical School. "I envision a future where pathologists have access to computational tools to assist with many tasks which are currently done manually. I think these technologies will enhance the practice of pathology."

Nevertheless, it's likely that more testing will be required before pathologists will revamp their standard procedures.

"Aside from the significant regulatory challenges in removing the physician from the diagnosis, the importance of clinical factors, tissue context and the inherent heterogeneity of cancer, and thus its diagnosis, will mean that computer assistance and imaging tools like these will remain an aid to pathologists for some time to come," said Steve Burnell, lifecycle leader for digital pathology and workflow at Roche Diagnostics.

One issue is that C-Path's calculations might have put pathologists at an unfair disadvantage. "The Stanford team had two separate groups of patient samples to work with but included a portion of one group to both train and test C-Path," said Ventana's Fernandez. "Ideally, one wants to use one group of samples to train and a separate group of samples to test" to avoid the potential of over-fitting the features.

Jared Schwartz, CMO at digital pathology company Aperio Technologies Inc., said the small tumor sample size used by C-Path could increase the odds of errors. "Tumors have great heterogeneity, and thus sampling issues are a major hurdle. The smaller the biopsies, the greater the sampling errors. Most pathologists would want to collect more than one biopsy sample per patient to get a complete look at the tumor."

But with the right samples, Schwartz added, C-Path "demonstrates that increasingly powerful tools will be available to enhance pathologists' ability to provide more and hopefully more precise diagnostic information to improve patient outcomes."

Koller agreed. C-Path's "performance is considerably better when we have more than one sample from each tumor," she said.

Burnell wanted Koller's team to show that C-Path can handle the inherent variability and more complex processing that comes from looking at a larger surface area of tumor tissue. 


\section{ANALYSIS}

Koller thinks looking at more tissue would actually improve C-Path's performance. "I would expect performance might get even better with whole-slide images, although we haven't really done that experiment," she said. "Moving from small tumor samples to whole-slide images is not a huge barrier."

Fernandez added that what he would like to see is more biological and pathological context extrapolated from the morphological features that C-Path uses to score samples. "They found clinically significant features based on outcome," he said. "Not all, however, had a sound pathological explanation. How do these features help explain pathologic grade and outcome in patients? What are the biological underpinnings?"

Michael Becich, chair of biomedical informatics at the University of Pittsburgh, was less interested in the biology and more focused on the "details about the machine learning used with C-Path. Perhaps as the IP is established, the team will provide more bioinformatics details."

The Stanford team is working on extending the method on wholetissue slide samples and hopes to set up a prospective, multicenter trial.
Ultimately, Koller would like C-Path to be able to use breast cancer sample data and predict which drugs would produce the best response in a given patient.

Baas, T. SciBX 4(48); doi:10.1038/scibx.2011.1342

Published online Dec. 15, 2011

\section{REFERENCES}

1. Beck, A.H. et al. Sci. Transl. Med.; published online Nov. 9, 2011; doi:10.1126/scitranslmed.3002564

Contact: Daphne Koller, Stanford University, Stanford, Calif. e-mail: koller@cs.stanford.edu

\section{COMPANIES AND INSTITUTIONS MENTIONED}

Aperio Technologies Inc., Vista, Calif.

Definiens AG, Munich, Germany

Harvard Medical School, Boston, Mass.

Roche (SIX:ROG; OTCQX:RHHBY), Basel, Switzerland

Stanford University, Stanford, Calif.

University of Pittsburgh, Pittsburgh, Pa.

Ventana Medical Systems Inc. (NASDAQ:VMSI), Tucson, Ariz. 